\title{
DIAGRAMAS DE INTERACCIÓN PARA SECCIONES METÁLICAS HUECAS A FLEXIÓN ESVIADA COMPUESTA
}

\author{
(INTERACTION GRAPHS FOR STEEL HOLLOW SECTIONS SUBJECTED TO AXIAL FORCE \\ AND BIAXIAL BENDING)
}

Ramón Irles Más, Dr. Ing. de Caminos, Prof. Tit. Univ.

$\mathrm{Dt}^{\circ}$. Ing. de la Construcción, OP e Inf. Urbana

Francisco Irles Más, ITOP, Prof. Tit. Int. EU

Fecha de recepción: $16-\mathrm{V}-94$

$\mathrm{Dt}^{\circ}$. Expresión Gráfica y Cartografía

$430-4$

Universidad de Alicante

Daniel Carratalá Climent, AT, Estudiante de $4 .{ }^{\circ}$ curso Arquitectura

Universidad Politécnica de Valencia

ESPAÑA

\begin{abstract}
RESUMEN
El uso de los diagramas de interacción como herramienta de predimensionado o dimensionado de secciones de piezas prismáticas sometidas a combinaciones de varios esfuerzos constituye algo habitual en la técnica. Desde hace tiempo se dispone de colecciones de estos diagramas para las diversas tecnologias: hormigón armado, acero estructural y secciones mixtas, bajo diversas solicitaciones.
\end{abstract}

En el artículo se expone minuciosamente el planteamiento general para la obtención de los diagramas correspondientes a flexión biaxil compuesta en secciones buecas, circulares y rectangulares o cuadradas, con o sin redondeo de vértices. En particular, se realiza un estudio general detallado de la superficie de interacción de estos tres esfuerzos en dichas secciones.

\section{SUMMARY}

Interaction graphs are extensively used as a tool for designing prismatic member sections subjected to several combined stresses. For a long time, sets of these graphs are available in building technologies: reinforced concrete, steel and composite sections, under various stresses.

The paper shows the general formulation for obtainning the graphs corresponding to biaxial bending with axial force of bollow steel sections; with circular, rectangular and square shape, with or without vertex aroundisment. In particular, a detailed general analysis of the interaction surface is made for these stresses over the above cited sections.

\section{Introducción}

La indiscutible comodidad de uso de los diagramas de interacción de varios esfuerzos combinados (hasta 3, generalmente), sobre una sección de pieza prismática, es la causa de la difusión de dichos grá- ficos. Entre ellos, cabe citar, como universales, los correspondientes a secciones de hormigón armado confeccionados por Jiménez Montoya y otros ${ }^{1}$; también pueden citarse los disponibles para perfilería metálica o mixta en varias publicaciones, entre las que cabe destacar los trabajos de Chen y otros ${ }^{2 y 3}$. 
En este artículo se presenta el planteamiento general que permite obtener analíticamente los diagramas de interacción axil-flexión biaxil en las secciones metálicas huecas habituales, para la situación en que plastifica el punto más solicitado. Se estudia el caso general de secciones rectangulares con vértices redondeados, que permite extender las conclusiones a los casos de vértices vivos, en un extremo, y al de sección circular en el otro, como particulares del caso general.

\section{Planteamiento general del problema}

Para una sección metálica rectangular hueca, con vértices redondeados (Fig. 1), sometida a flexión biaxil compuesta, el punto más solicitado siempre es uno del contorno circular exterior de una de las esquinas. El valor máximo es compartido por todos los de un lado del contorno exterior cuando la flexión es monoaxil.

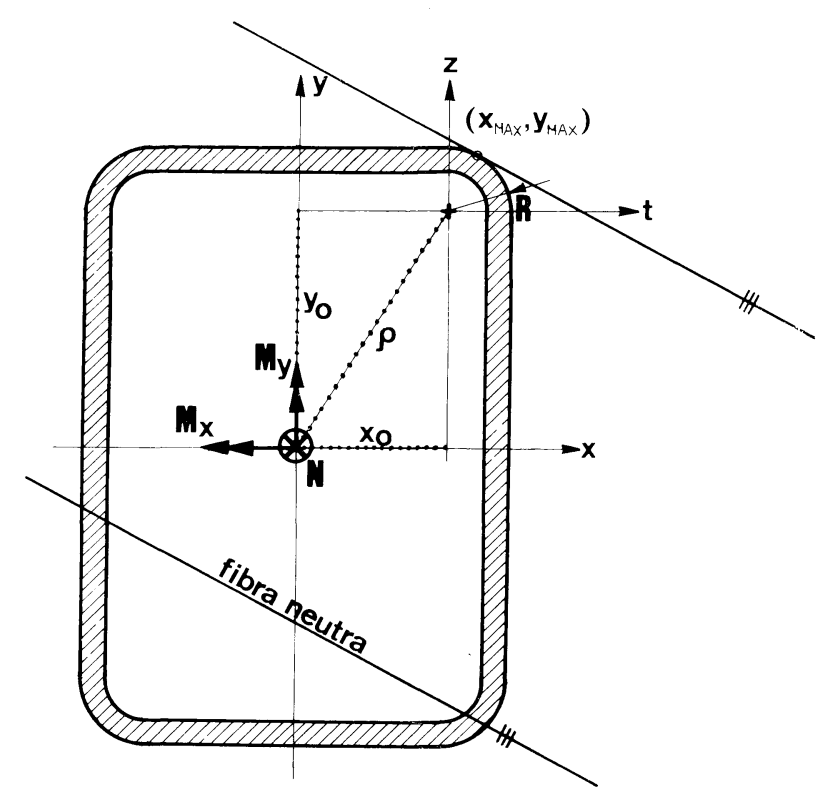

Fig. 1

Dada la doble simetría de estas secciones, bastará estudiar el cuadrante que presente las mayores tensiones, que será el más alejado de la fibra neutra. Por tanto, con la notación de dicha figura, tendremos:

Expresión general de las tensiones:

$$
\sigma(x, y)=\frac{N}{A}+\frac{M_{x}}{I_{x}} y+\frac{M_{y}}{I_{y}} x
$$

Que escribiremos así:

$$
\sigma(\mathrm{x}, \mathrm{y})=\nu+\mu_{\mathrm{x}} \mathrm{y}+\mu_{\mathrm{y}} \mathrm{x}
$$

Ecuación de la fibra neutra:

$$
\mathrm{O}=\nu+\mu_{\mathrm{x}} \mathrm{y}+\mu_{\mathrm{y}} \mathrm{x} ; \mathrm{y}_{\mathrm{FN}}=-\frac{\mu_{\mathrm{y}}}{\mu_{\mathrm{x}}} \mathrm{x}-\frac{v}{\mu_{\mathrm{x}}}
$$

Con el criterio, frecuente en estructura metálica, de admitir como solicitación máxima la combinación que hace alcanzar el límite elástico $\sigma_{\mathrm{e}}$ al punto de mayor tensión, la condición límite será:

$$
\sigma_{\mathrm{e}}=v+\mu_{\mathrm{x}} \mathrm{y}_{\max }+\mu_{\mathrm{y}} \mathrm{x}_{\max }
$$

Puesto que las tensiones [1] son planas, el punto de máxima tensión $\left(\mathrm{x}_{\max } \mathrm{y}_{\max }\right)$ será el más alejado de la fibra neutra, dentro de dicho contorno circular, es decir, aquel en el que la tangente al círculo sea paralela a la fibra neutra:

- Circunferencia

$$
\mathrm{z}=\sqrt{\mathrm{R}^{2}-\mathrm{t}^{2}}
$$

- El paralelismo exige:

$$
\mathrm{z}^{\prime}=\mathrm{y}_{\mathrm{FN}}^{\prime} \rightarrow \frac{\mathrm{t}_{\text {max }}}{\sqrt{\mathrm{R}^{2}-\mathrm{t}_{\text {max }}^{2}}}=\frac{\mu_{\mathrm{y}}}{\mu_{\mathrm{x}}}
$$

es decir:

$$
t_{\max }=\frac{R \mu_{y}}{\sqrt{\mu_{x}^{2}+\mu_{y}^{2}}}
$$

y de [5]:

$$
z_{\max }=\frac{R \mu_{x}}{\sqrt{\mu_{x}^{2}+\mu_{y}^{2}}}
$$

Y en los ejes centrales principales:

$$
x_{\max }=x_{0}+\frac{R \mu_{y}}{\sqrt{\mu_{x}^{2}+\mu_{y}^{2}}}
$$

$$
\mathrm{y}_{\max }=\mathrm{y}_{\mathrm{o}}+\frac{\mathrm{R} \mu_{\mathrm{x}}}{\sqrt{\mu_{\mathrm{x}}^{2}+\mu_{\mathrm{y}}^{2}}}
$$


Por lo tanto, la condición limite [4] será:

$\sigma_{\mathrm{e}}=\nu+\mu_{\mathrm{x}}\left(\mathrm{y}_{0}+\frac{\mathrm{R} \mu_{\mathrm{x}}}{\sqrt{\mu_{\mathrm{x}}^{2}+\mu_{\mathrm{y}}^{2}}}\right)+\mu_{\mathrm{y}}\left(\mathrm{x}_{0}+\frac{\mathrm{R} \mu_{\mathrm{y}}}{\sqrt{\mu_{\mathrm{x}}^{2}+\mu_{\mathrm{y}}^{2}}}\right)$

Después de racionalizar, elevar al cuadrado y ordenar, se cbtiene:

$$
\begin{aligned}
& \nu^{2}+\left(\mathrm{y}_{0}^{2}-\mathrm{R}^{2}\right) \mu_{\mathrm{x}}^{2}+\left(\mathrm{x}_{0}^{2}-\mathrm{R}^{2}\right) \mu_{\mathrm{y}}^{2}+2 \mathrm{y}_{0} \nu \mu_{\mathrm{x}}+2 \mathrm{x}_{0} \nu \mu_{\mathrm{y}}+ \\
& +2 \mathrm{x}_{0} \mathrm{y}_{0} \mu_{\mathrm{x}} \mu_{\mathrm{y}}-2 \sigma_{\mathrm{e}} \nu-2 \sigma_{\mathrm{e}} \mathrm{y}_{\mathrm{o}} \mu_{\mathrm{x}}-2 \sigma_{\mathrm{e}} \mathrm{x}_{0} \mu_{\mathrm{y}}+\sigma_{\mathrm{e}}^{2}=0
\end{aligned}
$$

ecuación de la superficie límite, que resulta ser una cuádrica en los ejes $\left(\nu, \mu_{\mathrm{x}}, \mu_{\mathrm{y}}\right)$, y también en los $N, M_{x}, M_{y}$.

\section{Estudio de la superficie límite}

\subsection{Caso general}

La expresión [7] representa una superficie cuádrica con ejes principales oblicuos a los $\nu, \mu_{x}, \mu_{y}$. A continuación, se procede a su estudio y clasificación.

Puesto que:

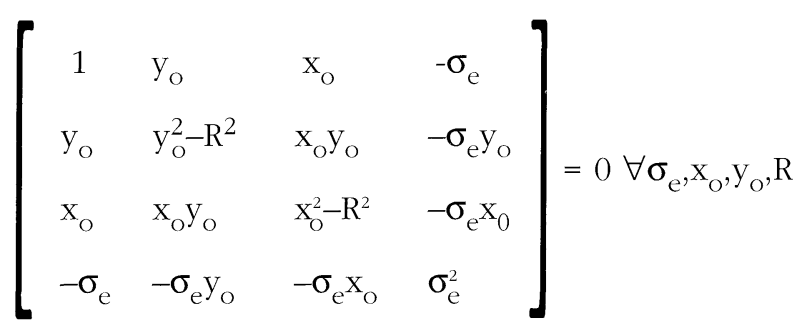

se trata, en todos los casos, de un cono.

Su centro $\left(v_{c}, \mu_{\mathrm{xc}}, \mu_{\mathrm{yc}}\right)$ es la solución de:

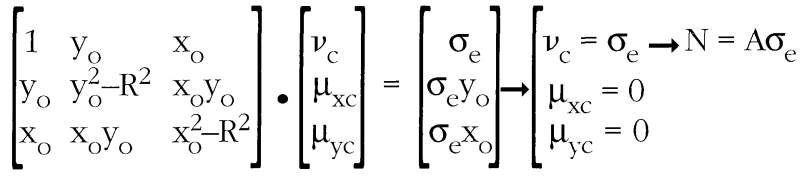

es decir, en todos los casos se encuentra sobre el eje $\mathrm{N}$, en el punto $N_{u}=A \sigma_{e}$.

Sus ejes principales son los autovectores correspondientes a los autovalores de esta matriz de coeficientes:

$$
\left[\begin{array}{lll}
1-\lambda & y_{O} & x_{O} \\
y_{O} & y_{O}^{2}-R^{2}-\lambda & x_{O} y_{O} \\
x_{O} & x_{O} y_{O} & x_{O}^{2}-R^{2}-\lambda
\end{array}\right]=0=\lambda^{3}+\lambda^{2}\left(2 R^{2}-1-\rho^{2}\right)+
$$

siendo $\rho^{2}=x_{0}^{2}+y_{0}^{2}$ (Fig. 1).
Las soluciones de esta ecuación son:

$$
\begin{gathered}
\lambda_{1}=\frac{1+\rho^{2}-R^{2}+\sqrt{\left(R^{2}-1-\rho^{2}\right)^{2}+4 R^{2}}}{2}>0 \\
\lambda_{2}=\frac{1+\rho^{2}-R^{2}-\sqrt{\left(R^{2}-1-\rho^{2}\right)^{2}+4 R^{2}}}{2}<0 \\
\lambda_{3}=-R^{2}<0
\end{gathered}
$$

y los correspondientes autovectores (sin normalizar) son:

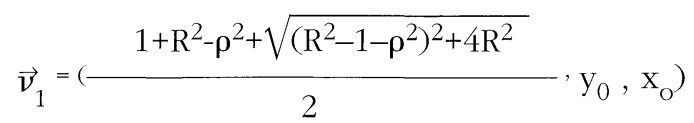

$$
\begin{gathered}
\vec{v}_{2}=\left(\frac{1+R^{2}-\rho^{2}-\sqrt{\left(R^{2}-1-\rho^{2}\right)^{2}+4 R^{2}}}{2}, y_{0}, x_{0}\right) \\
\vec{v}_{3}=\left(0,-x_{0}, y_{0}\right)
\end{gathered}
$$

Unos ejes $(m, n, \tilde{n})$ paralelos a estos vectores por el centro del cono $\left(A \sigma_{e}, 0,0\right)$ constituyen los principales de la cuádrica. El paralelo a $\vec{v}_{1}$ es el eje del cono, ya que $\lambda_{1}>0$. Efectuando un cambio de ejes a los principales (siendo $\mathrm{m}$ el eje del cono), la ecuación de la cuádrica se reduce a:

$$
\lambda_{1} \mathrm{~m}^{2}+\lambda_{2} \mathrm{n}^{2}+\lambda_{3} \tilde{\mathrm{n}}^{2}=0
$$

Lo que permite identificarlo, en general $\left(\lambda_{2} \neq \lambda_{3}\right)$, como un cono de directriz elíptica y eje $m$ oblicuo a los planos coordenados (Fig. 2), para el caso de secciones huecas rectangulares con vértices redondeados.

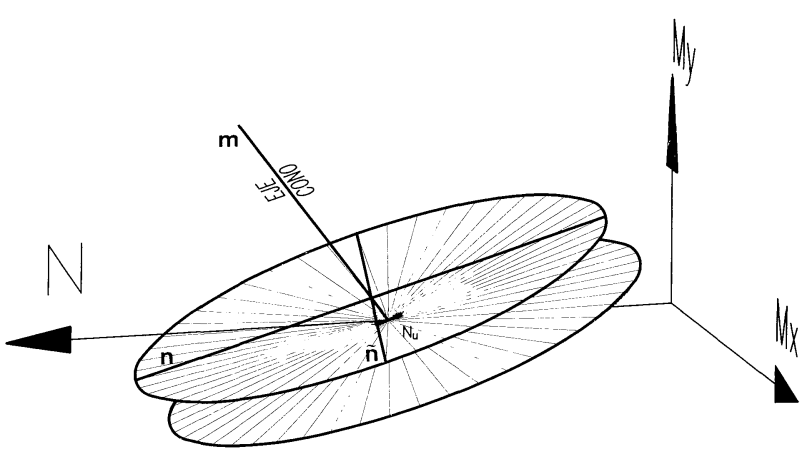




\subsection{Casos particulares}

\subsubsection{Sección rectangular sin redondeo de vértices}

En este caso, con $\mathrm{R}=0 \mathrm{y}\left(\mathrm{x}_{\max }, \mathrm{y}_{\max }\right)=\left(\mathrm{x}_{\mathrm{o}}, \mathrm{y}_{\mathrm{o}}\right)$, se obtiene:

$$
\lambda_{1}=1+\rho^{2} ; \quad \lambda_{2}=\lambda_{3}=0
$$

los semiángulos del cono valen $\pi / 2$, y éste degenera en un plano doble, de ecuación inmediata a partir de [1]:

$$
\sigma_{e}=\frac{N}{A}+\frac{M_{x}}{I_{x}} y_{O}+\frac{M_{y}}{I_{y}} x_{o}
$$

que pasa por los tres puntos $N_{\mathrm{u}}=\mathrm{A} \sigma_{\mathrm{e}}, \mathrm{M}_{\mathrm{xu}}=\sigma_{\mathrm{e}} \mathrm{I}_{\mathrm{x}} / \mathrm{y}_{\mathrm{o}}$, $M_{y u}=\sigma_{e} I_{y} / x_{o}$ (Fig. 3).

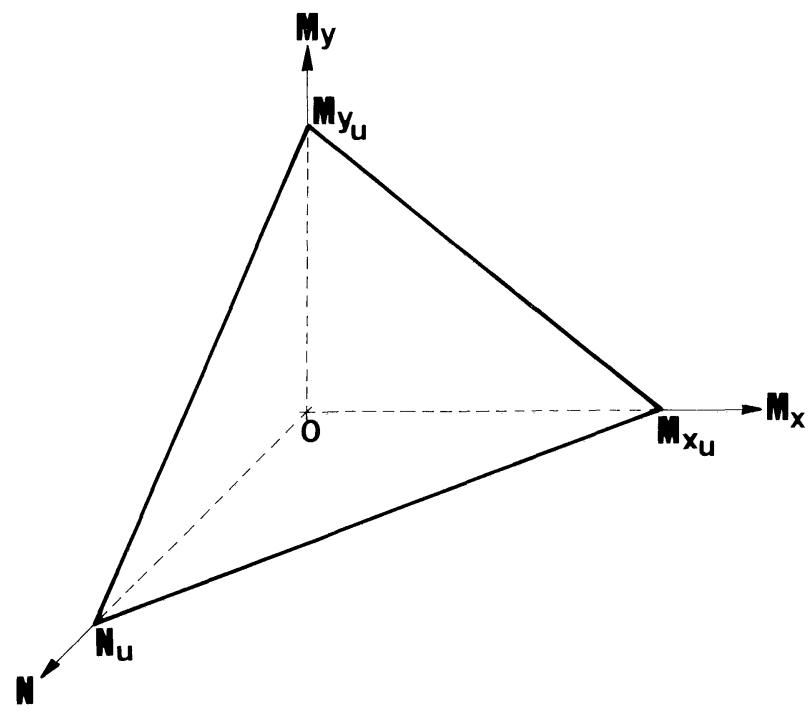

Fig. 3

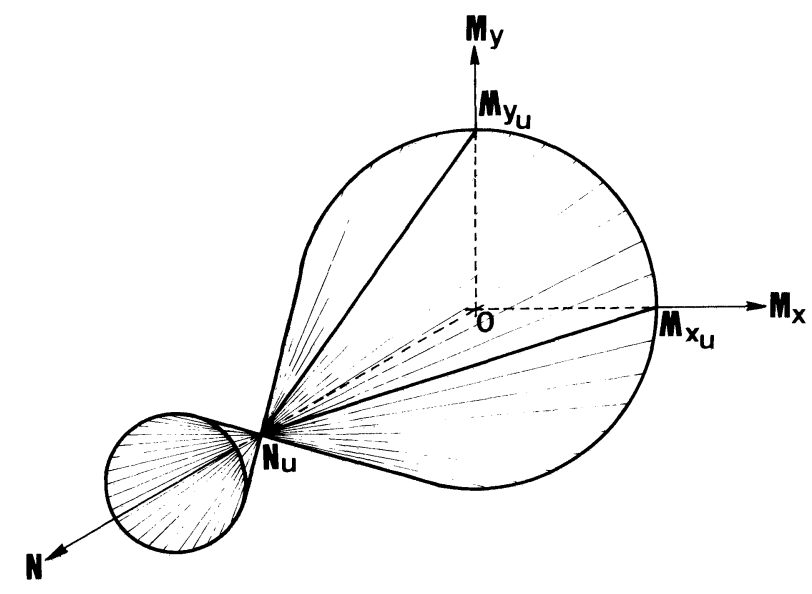

Fig. 4

\subsubsection{Sección cuadrada sin redondeo de vértices}

En este caso, la traza del plano que constituye la superficie de interacción con el $\mathrm{N}=0$ es paralela a su segunda bisectriz.

\subsubsection{Sección circular}

En este caso, $x_{0}=y_{0}=0=\rho$ y R es el radio exterior de la sección. Se obtiene:

$$
\lambda_{1}=1, \lambda_{2}=-\mathrm{R}^{2}, \lambda_{3}=-\mathrm{R}^{2}
$$

y el cono resulta de directriz circular. Su eje, oblicuo en el caso general a los planos coordenados, es ahora coincidente con el eje de axiles (Fig. 4), ya que el primer autovector es, ahora normalizado:

$$
\vec{v}_{1}=(1,0,0)
$$

\section{Representación de la superficie límite. Diagramas de interacción}

\subsection{Sección circular}

En la Fig. 5 se representa la porción de superficie de interacción contenida en el $1 .^{\text {er }}$ octante, que es la única a considerar tal como se ha planteado el problema. En ella se aprecia la realidad bidimensional del mismo en este caso, y la representación de la superficie puede reducirse a una recta en el plano $\mathrm{NOM}_{\mathrm{r}}$, siendo $\mathrm{M}_{\mathrm{r}}=\sqrt{\mathrm{M}_{\mathrm{x}}^{2}+\mathrm{M}_{\mathrm{y}}^{2}}$ el momento resultante; recta única que constituye el diagrama de interacción $\mathrm{N}-\mathrm{M}_{\mathrm{r}}$ de la sección circular correspondiente. En la Fig. 6 se representa el correspondiente a un perfil $\varnothing 100 \times 5\left(\sigma_{\mathrm{e}}=2.600\right)$.

Las líneas de nivel $\mathrm{N}=$ constante de la superficie son circunferencias concéntricas con el origen, que constituyen el diagrama de interacción en unos ejes arbitrarios, no coincidentes con el de momento resultante.

\subsection{Sección rectangular de vértices vivos}

En la Fig. 7 se representa la porción en 1.er octante del plano en el que degenera el caso general. En éste, el problema es tridimensional y todas las secciones planas son rectas paralelas para valores constantes de cualquiera de las tres variables. Representando, por ejemplo, las líneas de nivel $\mathrm{N}=$ constante de la superficie de interacción, se obtiene un diagrama como el de Fig. 8, que consti- 


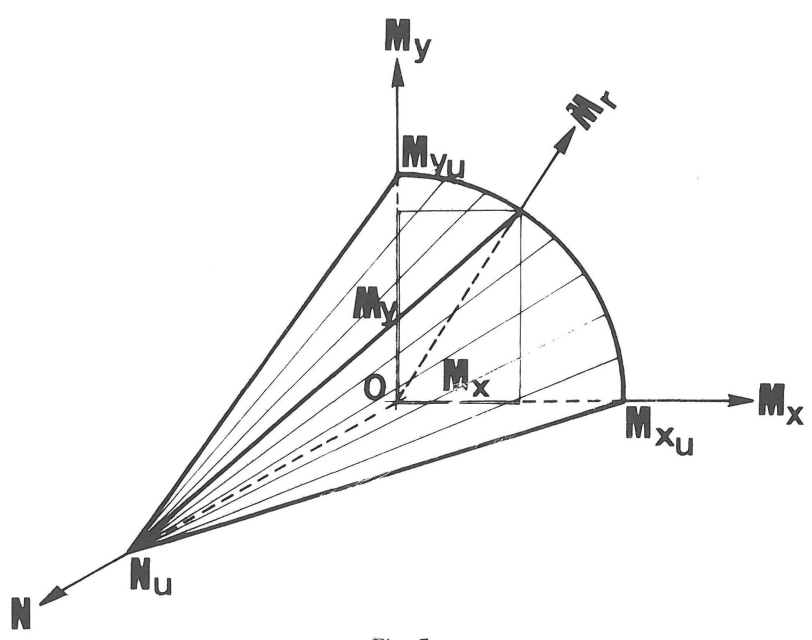

Fig. 5

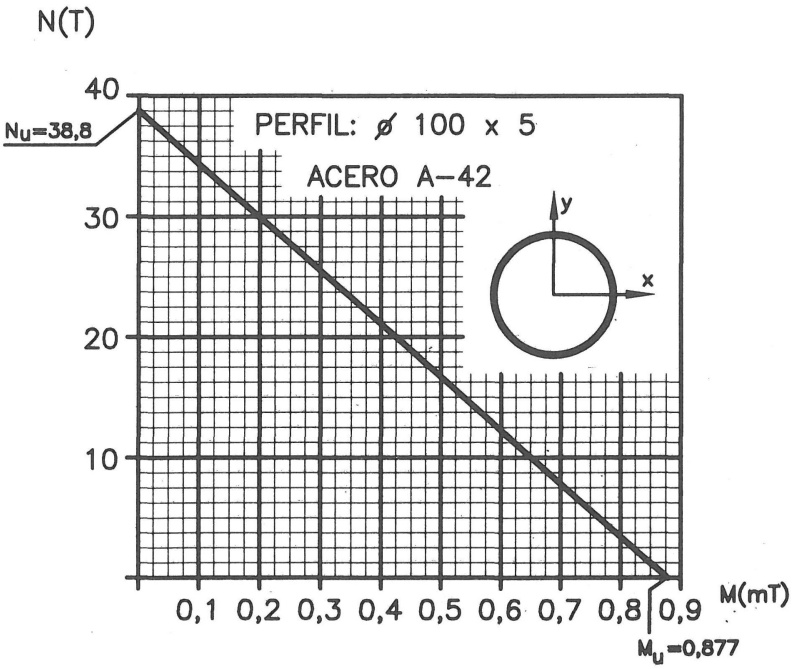

Fig. 6

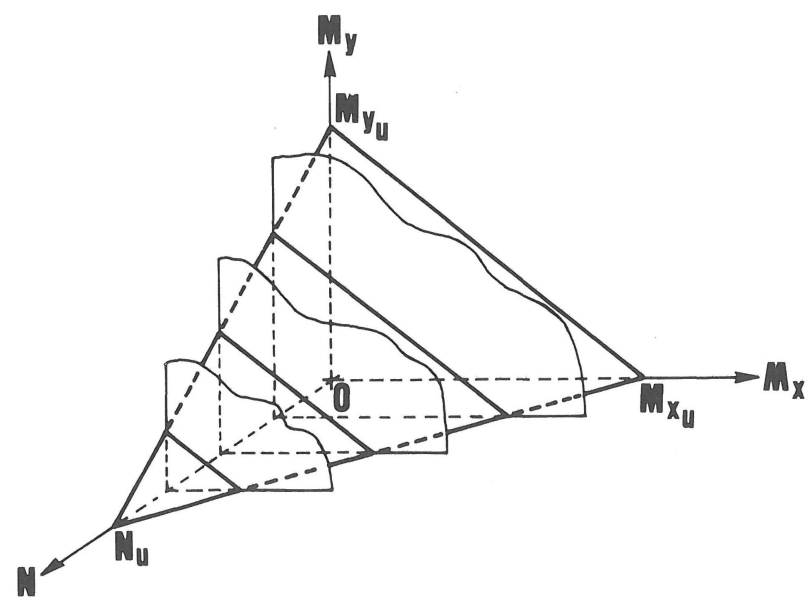

Fig. 7 tuye uno de los posibles de interacción $\mathrm{N}-\mathrm{M}_{\mathrm{x}}-\mathrm{M}_{\mathrm{y}}$ para este tipo de secciones.

Cuando la sección es cuadrada, y en la opción de cortar con planos $\mathrm{N}=$ constante, las rectas del diagrama de interacción son paralelas a la segunda bisectriz del plano $M_{x}-M_{y}$, dada la doble simetría de la sección.

En la Fig. 8 se representa el diagrama correspondiente a un \# $120 \times 60 \times 6$ de vértices vivos $\left(\sigma_{e}=\right.$ $=2.600)$.

\subsection{Sección rectangular con vértices redondeados}

Habida cuenta de la forma de la superficie de interacción en el caso general, las intersecciones con los planos paralelos a los coordenados son todas curvas cónicas, salvo las correspondientes al $\mathrm{NOM}_{\mathrm{x}}$ y $\mathrm{NOM}_{\mathrm{y}}$, que son rectas directrices, ya que el eje ON pasa por el centro del cono, ahora de eje oblicuo a dichos planos.

Si al igual que antes, se representa la superficie de interacción mediante sus curvas de nivel, $\nu=\mathrm{k}$ (lo cual constituye una de las opciones posibles, probablemente la más usual y conveniente), éstas resultan con una ecuación, a partir de [7]:

$$
\begin{gathered}
\mu_{\mathrm{x}}^{2}\left(\mathrm{y}_{0}^{2}-\mathrm{R}^{2}\right)+\mu_{\mathrm{y}}^{2}\left(\mathrm{x}_{0}^{2}-\mathrm{R}^{2}\right)+2\left(\mathrm{k}-\sigma_{\mathrm{e}}\right) \mathrm{y}_{0} \mu_{\mathrm{x}}+2\left(\mathrm{k}-\sigma_{\mathrm{e}}\right) \mathrm{x}_{0} \mu_{\mathrm{y}}+ \\
+2 \mathrm{x}_{0} \mathrm{y}_{0} \mu_{\mathrm{x}} \mu_{\mathrm{y}}+\left(\mathrm{k}-\sigma_{\mathrm{e}}\right)^{2}=0
\end{gathered}
$$

cónicas para cuya clasificación se obtienen a continuación los ejes principales:

$\left[\begin{array}{cc}y_{0}^{2}-R^{2-\lambda} & x_{0} y_{O} \\ x_{0} y_{O} & x_{0}^{2}-R^{2}-\lambda\end{array}\right]=0=\lambda^{2}+\lambda\left(2 R^{2}-\rho^{2}\right)+R^{2}\left(R^{2}-\rho^{2}\right)$

Autovalores: $\quad \lambda_{\mathrm{I}}=\rho^{2}-\mathrm{R}^{2}>0 \quad \lambda_{\mathrm{II}}=-\mathrm{R}^{2}<0$

Autovectores unitarios: $\vec{v}_{\mathrm{I}}=\left(\frac{\mathrm{y}_{0}}{\rho}, \frac{\mathrm{x}_{\mathrm{O}}}{\rho}\right){\overrightarrow{\mathrm{v}_{\mathrm{L}}}}_{\mathrm{L}}=\left(-\frac{\mathrm{x}_{\mathrm{O}}}{\rho}, \frac{\mathrm{y}_{\mathrm{O}}}{\rho}\right)$

realizando el cambio a los ejes $(\xi, \eta)$, dados por $\vec{v}_{\mathrm{I}}$, $\vec{v}_{\text {II }}$ resulta, en forma canónica:

$$
\frac{\left[\xi+\frac{\left(k-\sigma_{e}\right) \rho}{\rho^{2}-R^{2}}\right]^{2}}{\left[\frac{\left(k-\sigma_{e}\right) R}{\rho^{2}-R^{2}}\right]^{2}}-\frac{\eta^{2}}{\left[\frac{k-\sigma_{e}}{\sqrt{\rho^{2}-R^{2}}}\right]^{2}}=1
$$



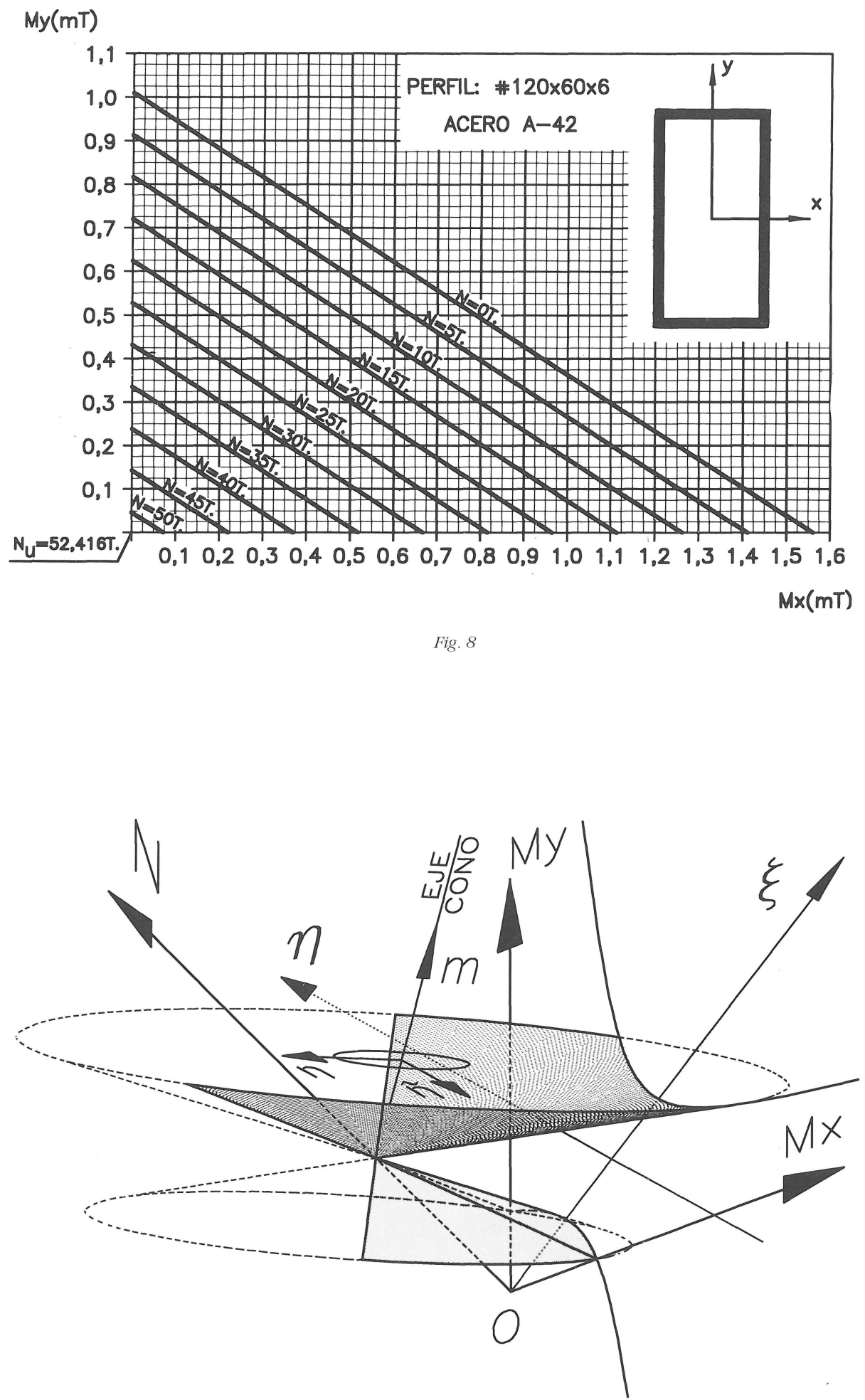

es decir, hipérbolas no equiláteras de centro $\left[\rho\left(\sigma_{e}-k\right) /\right.$ $\left./\left(\rho^{2}-R^{2}\right), 0\right]$ en los ejes $(\xi, \eta)$. La correspondiente a $\mathrm{N}=0$ se representa en la Fig. 9 sobre el plano $\mathrm{M}_{\mathrm{x}}{ }^{-}$ $-\mathrm{M}_{\mathrm{y}}$. Su rama más próxima al origen, correspondiente a la hoja del cono que se desarrolla sobre su semieje no incluido en el primer octante, corta a los ejes $\mathrm{OM}_{\mathrm{x}}$ y $\mathrm{OM}_{\mathrm{y}}$ en los puntos

$$
\mathrm{M}_{\mathrm{xu}}=\frac{\mathrm{I}_{\mathrm{x}} \sigma_{\mathrm{e}}}{\mathrm{y}_{\mathrm{o}}+\mathrm{R}} \quad \mathrm{M}_{\mathrm{yu}}=\frac{\mathrm{I}_{\mathrm{y}} \sigma_{\mathrm{e}}}{\mathrm{x}_{\mathrm{o}}+\mathrm{R}}
$$

La porción de esta rama comprendida entre dichos puntos es la única a considerar en el problema, pues la otra rama, correspondiente a la otra hoja del cono, proporciona valores fuera del rango del mismo. 


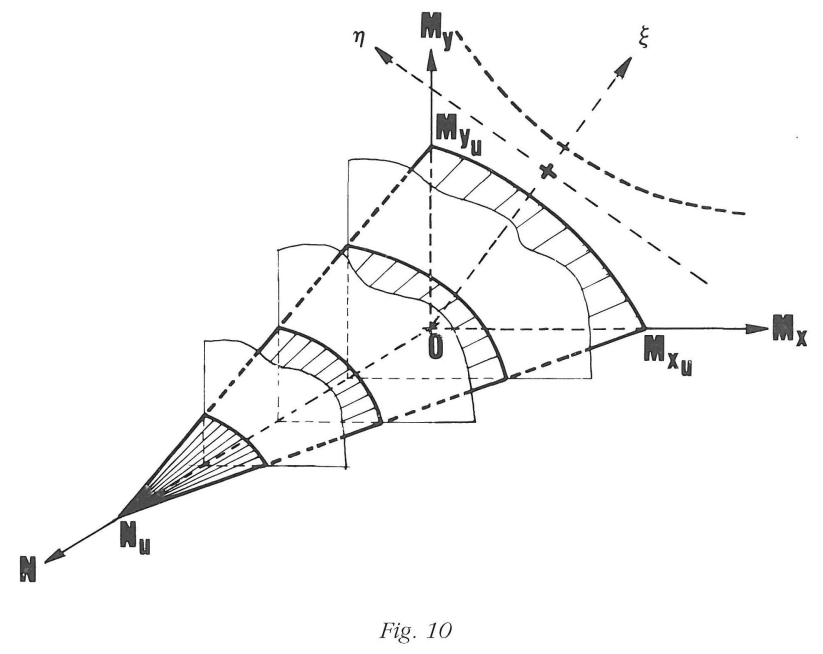

En la Fig. 10 se representa la generación de dichas curvas de nivel $\nu=\mathrm{k}$, y en la 11 puede verse el diagrama de interacción así generado para un perfil \# 120x60x6 de ENSIDESA $\left(\sigma_{\mathrm{e}}=2.600\right)$, ya en los ejes $\mathrm{N}-\mathrm{M}_{\mathrm{x}}-\mathrm{M}_{\mathrm{y}}$, preparado para su uso.

En el caso de perfiles cuadrados con vértices redondeados, todas estas consideraciones siguen válidas, y, además, el eje principal $\xi$ de la hipérbola coincide con la bisectriz del $1 .{ }^{\text {er }}$ cuadrante $M_{x}-M_{y}$, dada la doble simetría de la sección.

Nota: Como es evidente, los valores de $\mathrm{N}, \mathrm{M}_{\mathrm{x}}, \mathrm{M}_{\mathrm{y}}$ representados en los diagramas ya deben incluir los correspondientes coeficientes de ponderación, así como el de pandeo, en su caso.

\section{Conclusión}

En este artículo se ha estudiado de una forma general el aspecto de la superficie de interacción axil-flexión biaxil de los perfiles metálicos huecos habituales.

El enfoque del problema permite el estudio de todos los casos con una notable unidad, y la obtención concreta de los diagramas de interacción $\mathrm{N}-\mathrm{M}_{\mathrm{x}}$ $\mathrm{M}_{\mathrm{y}}$ para los distintos tipos de perfiles.

La superficie de interacción es siempre un cono, en dichos ejes.

En los perfiles rectangulares con vértices redondeados, el cono es de directriz elíptica con su eje oblicuo a los planos coordenados, y sus curvas de nivel $\mathrm{N}=$ constante resultan ser hipérbolas no equiláteras.

En los rectangulares con vértices vivos, el cono degenera en un plano doble y los diagramas de interacción están definidos por rectas paralelas.

Finalmente, en el caso de sección circular, el cono es de directriz circular, su eje el de axiles, y los diagramas de interacción trazados por curvas $\mathrm{N}=$ constante son circunferencias concéntricas; pero queda manifiesta la realidad bidimensional del problema, al ser indiferente la dirección de flexión resultante, pudiéndose reducir el diagrama de interacción a una sola recta en el plano Axil-Flexión total.

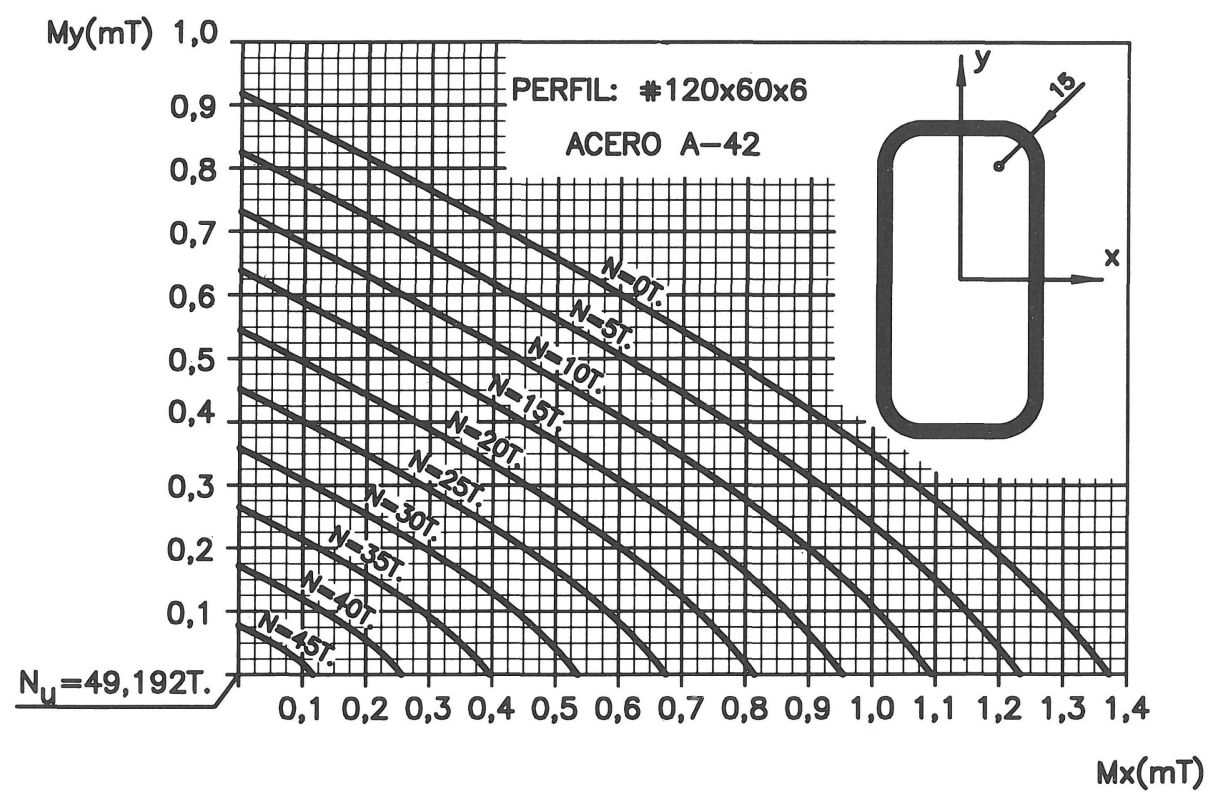


1. P. J. Montoya; A. G. Meseguer, y F. Morán. Gustavo Gili (1991): Hormigón Armado, Barcelona. Ed. 13, 1991.

2. S. P. Zhou, y W. F. Chen (1985): "Design Criteria for Box Columns under Biaxial Loading”. , Journal of structural Engrg, volumen 111, número 12, diciembre.
3. T. Atsuta, y W. F. Chen (1976): Theory of Beam-Columns, tomo $2 .^{\circ}$. McGraw-Hill B.C..

4 J. García García, y M. López Pellicer (1984): Álgebra lineal y Geometría, Marfil, Alcoy. 4. ${ }^{a}$ Ed.

\section{Publicación del Instituto Eduardo Torroja - CSIC}

\begin{tabular}{|c|c|}
\hline 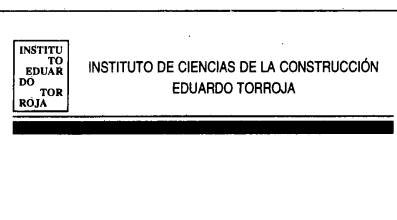 & $\begin{array}{l}\text { La revista MATERIALES DE CONSTRUCCIÓN, con periodicidad trimestral y dedicada } \\
\text { con exclusividad a la investigación científica y técnica de la construcción y sus materia- } \\
\text { les, está editada por el INSTITUTO EDUARDO TORROJA, Centro dependiente del } \\
\text { Consejo Superior de Investigaciones Científicas. Constituye, desde hace cuarenta y } \\
\text { cinco años, un eficaz vehículo de transmisión de nuevos conocimientos científicos y téc- } \\
\text { nicos en el campo de los materiales de construcción y de otros temas relacionados con } \\
\text { los mismos. }\end{array}$ \\
\hline & $\begin{array}{l}\text { Prestigiosos científicos y técnicos de todo el mundo colaboran en su publicación; no obstante, } \\
\text { MATERIALES ha pretendido tener siempre una especial incidencia en el mundo investigador } \\
\text { y técnico en el ámbito internacional y, muy especialmente, en los países de habla hispana. }\end{array}$ \\
\hline DE & $\begin{array}{l}\text { Conviene resaltar, una vez más, la importancia que tiene el desarrollo de los materiales } \\
\text { de construcción considerados tradicionales: el cemento y el hormigón, no sólo desde el } \\
\text { punto de vista técnico sino por la importancia social y económica que conlleva ese } \\
\text { desarrollo. Como muestra, se pueden recordar unas recientes declaraciones del doctor } \\
\text { Schartz, del Instituto Nacional de Normalización de Estados Unidos, en las que decía: } \\
\text { "La investigación del hormigón supone un enorme potencial de mejora en ese campo. } \\
\text { Lo que se haya de invertir en los próximos veinticinco años, puede depender de una } \\
\text { pequeña inversión en el quinquenio inmediato." }\end{array}$ \\
\hline 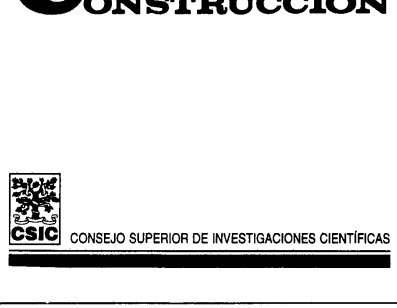 & $\begin{array}{l}\text { La indudable interrelación de disciplinas y ámbitos ha aconsejado ampliar la línea edito- } \\
\text { rial de MATERIALES a otros campos del conocimiento que no estaban suficientemente } \\
\text { recogidos. Por lo cual nuestra Revista incluye con mayor frecuencia artículos de investi- } \\
\text { gación -e incluso de divulgación-, temas cuya importancia está siendo objeto de una } \\
\text { atención prioritaria tanto por las autoridades científicas de nuestro país, como por los } \\
\text { propios responsables de la política investigadora europea. Un ejemplo destacado son } \\
\text { los estudios sobre Restauración y Conservación del Patrimonio Histórico y, en general, } \\
\text { del Patrimonio Arquitectónico y de la incidencia del medio ambiente en el comporta- } \\
\text { miento de los materiales, tanto tradicionales como no tradicionales. Algunos de estos } \\
\text { artículos se publican en versión española e inglesa. }\end{array}$ \\
\hline
\end{tabular}

